\title{
HAAR SYSTEMS ON EQUIVALENT GROUPOIDS
}

\author{
DANA P. WILLIAMS
}

(Communicated by Marius Junge)

\begin{abstract}
For second countable locally compact Hausdorff groupoids, the property of possessing a Haar system is preserved by equivalence.
\end{abstract}

\section{INTRODUCTION}

Beginning with the publication of Renault's seminal paper [11, locally compact groupoids have been an especially important way to construct operator algebras. Just as with the time honored group $C^{*}$-algebra construction, this is done by turning $C_{c}(G)$ into a convolution algebra and then completing. In the group case, there is always a (left) Haar measure on $G$ which allows us to form the convolution product. In the groupoid case, the natural convolution formula requires a family of measures $\lambda^{u}$ with support $G^{u}=\{x \in G: r(x)=u\}$ for each $u \in G^{(0)}$. We want the family to be left-invariant in that $x \cdot \lambda^{s(x)}=\lambda^{r(x)}$ where $x \cdot \lambda^{s(x)}(E)=\lambda^{s(x)}\left(x^{-1} E\right)$. In order that the convolution formula return a continuous function, we need the continuity condition that

$$
u \mapsto \int_{G} f(x) d \lambda^{u}(x)
$$

is continuous for all $f \in C_{c}(G)$ (the necessity is the main result in 15). Such a family $\left\{\lambda^{u}\right\}_{u \in G^{(0)}}$ is called a (continuous) Haar system for $G$. An annoying gap in the theory is that there is no theorem guaranteeing that Haar systems exist. The only significant positive existence result we are aware of is that if $G^{(0)}$ is open in $G$ and the range map is open (and hence the source map as well), then the family consisting of counting measures is always a Haar system. Groupoids with $G^{(0)}$ open and for which the range map is open are called étale. (It is also true that Lie groupoids necessarily have Haar systems [9, Theorem 2.3.1], but this result is crucially dependent on the manifold structure and hence is not in the spirit of this note.) It is also well known that if $G$ is any locally compact groupoid with a Haar system, then its range and source maps must be open. (This is a consequence of Remark 2.2 and Lemma 2.3.) Thus if a locally compact groupoid has a range map which is not open, then it can't possess a Haar system. Such groupoids do exist; for example, see [15, §3]. However, to the best of our knowledge, there is no example of a locally compact groupoid with open range and source maps which does not

Received by the editors January 16, 2015 and, in revised form, May 12, 2015.

2010 Mathematics Subject Classification. Primary 22A22; Secondary 28C15, 46L55, 46L05.

Key words and phrases. Groupoid, equivalent groupoids, Haar system, imprimitivity groupoid, $\pi$-system, proper action.

This work was supported in part by a grant from the Simons Foundation. 
possess a Haar system. We have yet to find an expert willing to conjecture (even off the record) that all such groupoids need have Haar systems, but the question remains open.

The purpose of this paper is to provide some additional examples where Haar systems must exist. The main result being that if $G$ and $H$ are equivalent second countable locally compact groupoids (as defined in [7] for example), and if $G$ has a Haar system, then so does $H$. Since equivalence is such a powerful tool, this result gives the existence of Haar systems on a great number of interesting groupoids. For example, every transitive groupoid with open range and source maps has a Haar system (Proposition 3.4).

The proof given here depends on several significant results from the literature. The first is that if $\pi: Y \rightarrow X$ is a continuous, open surjection with $Y$ second countable, then there is a family of Radon measures $\left\{\beta^{x}\right\}$ on $Y$ such that $\operatorname{supp} \beta^{x}=$ $\pi^{-1}(x)$ and

$$
x \mapsto \int_{Y} f(y) d \beta^{x}(y)
$$

is continuous for all $f \in C_{c}(Y)$. (This result is due to Blanchard, who makes use of a theorem of Michael [6].) The second is the characterization in [5, Proposition 5.2] of when the imprimitivity groupoid of a free and proper $G$-space has a Haar system. The third is the concept of a Bruhat section or cut-off function. These are used in [3. Chapter 7] to construct invariant measures. They also appear prominently in [13, Lemma 25] and [16, §6].

Since Blanchard's result requires separability, we can only consider second countable groupoids here.

\section{THE THEOREM}

Theorem 2.1. Suppose that $G$ is a second countable, locally compact Hausdorff groupoid with a Haar system $\left\{\lambda^{u}\right\}_{u \in G^{(0)}}$. If $H$ is a second countable, locally compact groupoid which is equivalent to $G$, then $H$ has a Haar system.

As in [12, p. 69] or [1, Definition 1.1.1], if $\pi: Y \rightarrow X$ is a continuous map between locally compact spaces $Y$ and $X$, then a $\pi$-system is a family of (positive) Radon measures $\left\{\beta^{x}: x \in X\right\}$ on $Y$ such that $\operatorname{supp} \beta^{x} \subset \pi^{-1}(x)$ and for every $f \in C_{c}(Y)$, the function

$$
x \mapsto \int_{Y} f(y) d \beta^{x}(y)
$$

is continuous. We say that $\beta$ is full if $\operatorname{supp} \beta^{x}=\pi^{-1}(x)$ for all $x \in X$.

If $Y$ and $X$ are both (left) $G$-spaces and $\pi$ is equivariant, then we say $\beta$ is equivariant if $\gamma \cdot \beta^{x}=\beta^{\gamma \cdot x}$, where $\gamma \cdot \beta^{x}(E)=\beta^{x}\left(\gamma^{-1} \cdot E\right)$ for all $(\gamma, x) \in G * X=$ $\{(\gamma, x): s(\gamma)=r(x)\}$. Alternatively,

$$
\int_{Y} f(\gamma \cdot y) d \beta^{x}(y)=\int_{Y} f(y) d \beta^{\gamma \cdot x}(y)
$$

for all $f \in C_{c}(Y)$ and $(\gamma, x) \in G * X$.

Remark 2.2. It is useful to keep in mind that a Haar system on $G$ is a full, equivariant $r$-system on $G$ for the range map $r: G \rightarrow G^{(0)}$.

In many cases, such as [5, $\S 5], \pi$-systems are reserved for continuous and open maps $\pi: Y \rightarrow X$. In the case of full systems, the next lemma implies that there is 
no loss in generality. (This part of the result does not require second countability.) Conversely, if $Y$ is second countable and $\pi$ is an open surjection, then Blanchard has proved that full $\pi$ systems must exist. Blanchard's result will be crucial in the proof of the main result.

Lemma 2.3 (Blanchard). Suppose that $\pi: Y \rightarrow X$ is a continuous surjection between second countable locally compact Hausdorff spaces. Then $\pi$ is open if and only if it admits a full $\pi$ system.

Proof. Suppose that $\beta$ is a full $\pi$ system. Let $V \subset Y$ be a nonempty open subset. Fix $x_{0} \in \pi(V)$ and $y_{0} \in V$ such that $\pi\left(y_{0}\right)=x_{0}$. Choose $U$ open such that $y_{0} \in U \subset \bar{U} \subset V$ and $\bar{U}$ is compact. Let $f \in C_{c}^{+}(Y)$ such that $f\left(y_{0}\right)=1$ and $\operatorname{supp} f \subset U$. Since $\beta$ is full, $\beta(f)\left(x_{0}\right)>0$. But $\beta(f)$ is continuous and $\operatorname{supp} \beta(f) \subset \pi(\bar{U}) \subset \pi(V)$. Hence $x_{0}$ is an interior point of $\pi(V)$. Since $x_{0}$ was arbitrary, $\pi(V)$ is open.

The converse is much more subtle and is due to Blanchard. The proof can be extracted from [2, §3.1] as follows. Note that the openness of $\pi$ implies that $C_{0}(Y)$ is a continuous field of $C^{*}$-algebras over $X$ [18, Theorem C.26]. Now we can apply [2, Proposition 3.9] thanks to [2, Proposition 3.5] 1]

We also need what is sometimes called a Bruhat section or cut-off function for $\pi$. The construction is modeled after Lemma 1 in Appendix I of [3, Chapter 7]. Recall that a subset $A \subset Y$ is called $\pi$-compact if $A \cap \pi^{-1}(K)$ is compact whenever $K$ is compact in $X$. We write $C_{c, \pi}(Y)$ for the set of continuous functions on $Y$ with $\pi$-compact support.

Lemma 2.4. Let $\pi: Y \rightarrow X$ be a continuous open surjection between second countable locally compact Hausdorff spaces. Then there is a $\varphi \in C_{c, \pi}^{+}(Y)$ such that $\pi(\{y \in Y: \varphi(y)>0\})=X$.

Proof. Let $\mathscr{V}=\left\{V_{i}\right\}$ be a countable, locally finite cover of $X$ by pre-compact open sets $V_{i}$. Let $\left\{\alpha_{i}\right\}$ be a partition of unity on $X$ subordinate to $\mathscr{V}$. Let $\varphi_{i} \in C_{c}^{+}(Y)$ be such that $\pi\left(\left\{y \in Y: \varphi_{i}>0\right\}\right) \supset V_{i}$. Then we can define

$$
\varphi(y)=\sum_{i} \varphi_{i}(y) \alpha_{i}(\pi(y))
$$

Since $\mathscr{V}$ is locally finite, the above sum is finite in a neighborhood of any $y \in Y$. Hence $\varphi$ is well defined and continuous. Local finiteness also implies that every compact subset of $X$ meets at most finitely many $V_{i}$. Since $\left\{\alpha_{i}\right\}$ is subordinate to $\mathscr{V}$, it follows that $\varphi$ has $\pi$-compact support. If $x \in X$, then there is an $i$ such that $\alpha_{i}(x)>0$. Then there is a $y$ such that $\varphi_{i}(y)>0$ and $\pi(y)=x$. Hence the result.

Proof of Theorem 2.1. Let $Z$ be a $(G, H)$-equivalence. Then the opposite module, $Z^{\mathrm{op}}$, is an $(H, G)$-equivalence. Therefore, in view of [5, Proposition 5.2], it will

\footnotetext{
${ }^{1}$ There is a slight gap in the proof of [2, Lemme 3.8]: it is asserted there that if $A$ is a separable $C^{*}$-algebra, then its dual $A^{*}$, equipped with the topology of uniform convergence on compact subsets of $A$, is a Fréchet space. However, if $A$ is infinite dimensional, then $A^{*}$ is not first countable in this topology. Unfortunately, this invalidates the application of [2 Lemme 3.6] in the proof of [2, Proposition 3.9]. Fortunately, 2, Lemme 3.6] can be modified (using the notation of [2] Lemme 3.6]) to the case where the topological vector space $E$ contains a complete convex subset $B$ containing the domain $T$. Thus the main results of [2, §3] remain true as stated.
} 


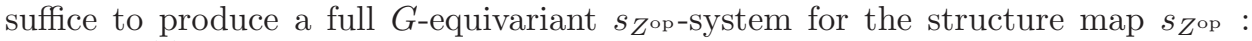
$Z^{\mathrm{op}} \rightarrow G^{(0)}$. Equivalently, we need a full equivariant $r_{Z}$-system for the map $r_{Z}$ : $Z \rightarrow G^{(0)} 2$ Hence the main theorem is a consequence of Proposition 2.5 below.

The following proposition is even more than what is called for in the proof of Theorem 2.1 it shows that every proper $G$-space has a full equivariant $r$-system for the moment map whether the action is free or not 3 It should be noted that pairs $(X, \alpha)$ where $X$ is a proper $G$-space and $\alpha$ and equivariant $r$-system play an important role in the constructions in [12] and [4. This makes even more interesting the assertion that such $\alpha$ 's always exist.

Proposition 2.5. Let $G$ be a second countable locally compact Hausdorff groupoid with a Haar system $\left\{\lambda^{u}\right\}_{u \in G^{(0)}}$. Suppose that $Z$ is a proper $G$-space with open momment map $r_{Z}: Z \rightarrow G^{(0)}$. Then there is a full equivariant $r_{Z^{-}}$system $\left\{\nu^{u}\right\}_{u \in G^{(0)}}$.

Blanchard's Lemma 2.3 implies that there is a full $r_{Z^{-}}$system $\beta=\left\{\beta^{u}\right\}_{u \in G^{(0)}}$. The idea of the proof of Proposition 2.5 is to use the Haar system on $G$ to average this system to create an equivariant system. The technicalities are provided by the next lemma. Notice that since $G$ acts properly, the orbit map $q: Z \rightarrow G \backslash Z$ is a continuous open surjection between locally compact Hausdorff spaces.

Lemma 2.6. Let $G, Z, \lambda$ and $\beta$ be as above.

(a) If $F \in C_{c}(G \times Z)$, then

$$
\Phi(F)(g, u)=\int_{Z} F(g, z) d \beta^{u}(z)
$$

defines an element of $C_{c}\left(G \times G^{(0)}\right)$.

(b) If $f \in C_{c}(Z)$ and $\varphi \in C_{c, q}(Z)$, then

$$
\Psi_{\varphi}(f)(g)=\int_{Z} f(g \cdot z) \varphi(z) d \beta^{s(g)}(z)
$$

defines an element of $C_{c}(G)$.

Proof. (a) This is straightforward if $F(g, z)=f(g) \varphi(z)$ with $f \in C_{c}(G)$ and $\varphi \in$ $C_{c}(Z)$. But we can approximate $F$ in the inductive limit topology with sums of such functions.

(b) Let $L=\operatorname{supp} \varphi \cap q^{-1}(q(\operatorname{supp} f))$. By assumption on $\varphi, L$ is compact. Since $G$ acts properly on $Z$, the set

$$
P(\operatorname{supp} f, L)=\{g \in G: g \cdot L \cap \operatorname{supp} f \neq \emptyset\}
$$

is compact. It follows that

$$
F(g, z)=f(g \cdot z) \varphi(z)
$$

defines an element of $C_{c}(G \times Z)$. Then

$$
\Psi_{\varphi}(f)(g)=\Phi(F)(g, s(g)) .
$$

The assertion follows.

\footnotetext{
${ }^{2}$ The gymnastics with the opposite space is just to accommodate a preference for left-actions. This has the advantage of making closer contact with the literature on $\pi$-systems.

${ }^{3}$ The literature is inconsistent as to whether the moment map for a groupoid action need be open. In this paper, it is critical that we use the definition of groupoid action that requires moment maps to be open.
} 
Proof of Proposition 2.5. Using Lemma 2.4 we fix $\varphi \in C_{c, q}^{+}(Z)$ such that $q(\{z: \varphi(z)>0\})=G \backslash Z$. Then we define a Radon measure on $C_{c}(Z)$ by

$$
\nu^{u}(f)=\int_{G} \int_{Z} f(g \cdot z) \varphi(z) d \beta^{s(g)}(z) d \lambda^{u}(g)=\int_{G} \Psi_{\varphi}(f)(g) d \lambda^{u}(g) .
$$

Since $\lambda$ is a Haar system and $\Psi_{\varphi}(f) \in C_{c}(G)$, we see immediately that

$$
u \mapsto \nu^{u}(f)
$$

is continuous.

Clearly, $\operatorname{supp} \nu^{u} \subset r_{Z}^{-1}(u)$. Suppose $r_{Z}(w)=u$ and $f \in C_{c}^{+}(Z)$ is such that $f(w)>0$. Then there is a $z^{\prime} \in\{z: \varphi(z)>0\}$ such that $q\left(z^{\prime}\right)=q(w)$. Hence there is a $g \in G$ such that $g \cdot z^{\prime}=w$. Note that $r_{Z}\left(z^{\prime}\right)=s(g)$ and $r(g)=r_{Z}(g \cdot z)=$ $r_{Z}(w)=u$. Since $\beta^{s(g)}$ has full support and since everything in sight is continuous and nonnegative,

$$
\Psi_{\varphi}(g)=\int_{Z} f(g \cdot z) \varphi(z) \beta^{s(g)}(z)>0 .
$$

Hence $\nu^{u}(f)>0$ and we have

$$
\operatorname{supp} \nu^{u}=r_{Z}^{-1}(u)
$$

Hence to complete the proof of the theorem, we just need to establish equivariance. But

$$
\begin{aligned}
\int_{Z} f\left(g^{\prime} \cdot z\right) d \nu^{s\left(g^{\prime}\right)}(z) & =\int_{G} \int_{Z} f\left(g^{\prime} g \cdot z\right) \varphi(z) d \beta^{s(g)}(z) d \lambda^{s\left(g^{\prime}\right)}(g) \\
& =\int_{G} \Psi_{\varphi}(f)\left(g^{\prime} g\right) d \lambda^{s\left(g^{\prime}\right)}(g),
\end{aligned}
$$

which, since $\lambda$ is a Haar system on $G$, is

$$
\begin{aligned}
& =\int_{G} \Psi_{\varphi}(g) d \lambda^{r\left(g^{\prime}\right)}(g) \\
& =\int_{G} \int_{Z} f(g \cdot z) \varphi(z) d \beta^{s(g)}(z) d \lambda^{r\left(g^{\prime}\right)}(g) .
\end{aligned}
$$

which, since $g^{\prime} \cdot s\left(g^{\prime}\right)=r\left(g^{\prime}\right)$, is

$$
=\int_{Z} f(z) d \nu^{g^{\prime} \cdot s\left(g^{\prime}\right)}(z)
$$

This completes the proof.

Proposition 2.5 is interesting even for a group action. The result itself is no doubt known to experts, but is amusing nonetheless.

Corollary 2.7. Suppose that $G$ is a locally compact group acting properly on a space $X$. Then $X$ has at least one invariant measure with full support.

\section{EXAMPles AND COMMENTS}

Recall that a topological groupoid $G$ is étale if and only if the range map $r$ : $G \rightarrow G^{(0)}$ is a local homeomorphism. (Since inversion is a homeomorphism, $s$ : $G \rightarrow G^{(0)}$ is a local homeomorphism if and only if $r$ is as well.) As pointed out in the introduction, any étale groupoid $G$ has a Haar system. As a consequence of Theorem 2.1. any second countable groupoid equivalent to $G$ has a Haar system (provided $G$ is second countable). In this section, we want to look at some additional 
examples. In some cases it is possible and enlightening to describe the Haar system in finer detail.

3.1. Proper principal groupoids. Recall that $G$ is called principal if the natural action of $G$ on $G^{(0)}$ given by $x \cdot s(x)=r(x)$ is free. We call $G$ proper if this action is proper in that $(x, s(x)) \mapsto(r(x), s(x))$ is proper from $G \times G^{(0)} \rightarrow G^{(0)} \times G^{(0)}$. If $G$ is a proper principal groupoid with open range and source maps, then the orbit space $G \backslash G^{(0)}$ is locally compact Hausdorff, and it is straightforward to check that $G^{(0)}$ implements an equivalence between $G$ and the orbit space $G \backslash G^{(0)}$. Since the orbit space clearly has a Haar system, the following is a simple corollary of Theorem 2.1

Proposition 3.1 (Blanchard). Every second countable proper principal groupoid with open range and source maps has a Haar system.

Remark 3.2. If $G$ is a second countable proper principal groupoid with open range and source maps, then the orbit map $q: G^{(0)} \rightarrow G \backslash G^{(0)}$ sending $u$ to $\dot{u}$ is continuous and open. Hence Blanchard's Lemma 2.3 implies there is a full $q$-system $\left\{\beta^{\dot{u}}\right\}_{\dot{u} \in G \backslash G^{(0)}}$. It is not hard to check that $\lambda^{u}=\delta_{u} \times \beta^{\dot{u}}$ is a Haar system for

$$
G_{q}:=\left\{(u, v) \in G^{(0)} \times G^{(0)}: \dot{u}=\dot{v}\right\} .
$$

Since $x \mapsto(r(x), s(x))$ is a groupoid isomorphism of $G$ and $G_{q}$, we get an elementary description for a Haar system on $G$.

While there certainly exist groupoids that fail to have open range and source maps - and hence cannot have Haar systems - most of these examples are far from proper and principal. In fact the examples we've seen are all group bundles which are as a far from principal as possible. This poses an interesting question.

Question 3.3. Must a second countable, locally compact, proper principal groupoid have open range and source maps?

3.2. Transitive groupoids. Recall that a groupoid is called transitive if the natural action of $G$ on $G^{(0)}$ given by $x \cdot s(x):=r(x)$ is transitive. If $G$ is transitive and has open range and source maps, then $G$ is equivalent to any of its stability groups $H=G_{v}^{v}=\{x \in G: r(x)=v=s(x)\}$ for $v \in G^{(0)}$; the equivalence is given by $G_{v}$ with the obvious left $G$-action and right $H$-action. Second countability is required to see that the restriction of the range map to $G_{v}$ onto $G^{(0)}$ is open 4 Since locally compact groups always have a Haar measure, the following is an immediate consequence of Theorem 2.1. (Similar assertions can be found in [14.)

Proposition 3.4 (Seda). If $G$ is a second countable, locally compact transitive groupoid with open range and source maps, then $G$ has a Haar system.

As before, we don't know the answer to the following.

Question 3.5. Must a second countable, locally compact, transitive groupoid have open range and source maps?

\footnotetext{
${ }^{4}$ Proving the openness of $\left.r\right|_{G_{v}}$ is nontrivial. It follows from 10, Theorem 2.1] or Theorems $2.2 \mathrm{~A}$ and $2.2 \mathrm{~B}$ in [7]. The assertion and equivalence fail without the second countability assumption as observed in 7, Example 2.2].

It should be noted that in both [10] and 7] openness of the range and source maps on a topological groupoid is a standing assumption.
} 
3.3. Blowing up the unit space. While there are myriad ways that groupoid equivalences arise in applications, one standard technique deserves special mention (see [17 for example). Suppose that $G$ is a second countable locally compact groupoid with a Haar system (or at least open range and source maps). Let $f$ : $Z \rightarrow G^{(0)}$ be a continuous and open map. Then we can form the groupoid

$$
G[Z]=\{(z, g, w) \in Z \times G \times Z: f(z)=r(g) \text { and } s(g)=f(w)\} .
$$

(The operations are as expected: $\left(z^{\prime}, g^{\prime}, z\right)(z, g, w)=\left(z^{\prime}, g^{\prime} g, w\right)$ and $(z, g, w)^{-1}=$ $\left(w, g^{-1}, z\right)$.) The idea is that we use $f$ to "blow-up" the unit space of $G$ to all of $Z$. If $\varphi: G[Z] \rightarrow G$ is the homormorphism $(z, g, w) \mapsto g$, then we get a $(G[Z], G)$ equivalence given by "the graph of $\varphi$ " (see [5, §6]):

$$
W=\{(z, g) \in Z \times G: f(z)=r(g)\} .
$$

The left $G[Z]$-action is given by $(z, g, w) \cdot\left(w, g^{\prime}\right)=\left(z, g g^{\prime}\right)$ and the right $G$-action by $\left(w, g^{\prime}\right) \cdot g=\left(w, g^{\prime} g\right)$. The openness of the range map for $G$ is required to see that the structure map $r_{W}: W \rightarrow Z$ is open, while the openness of $f$ is required to see that $s_{W}: W \rightarrow G^{(0)}$ is open. Assuming $G$ has a Haar system and $Z$ is second countable, Theorem 2.1 implies that $G[Z]$ has a Haar system. However in this case we can do a bit better and write a tidy formula for the Haar system on the blow-up. We still require Blanchard's Lemma 2.3 where there is a full $f$-system for any continuous open map $f: Z \rightarrow G^{(0)}$ (provided that $Z$ is second countable).

Proposition 3.6. Suppose that $G$ is a locally compact Hausdorff groupoid with a Haar system $\left\{\lambda^{u}\right\}_{u \in G^{(0)}}$, and that $Z$ is second countable. Let $f: Z \rightarrow G^{(0)}$ be a continuous open map, and let

$$
G[Z]=\{(w, g, z) \in Z \times G \times Z: f(w)=r(g) \text { and } s(g)=f(z)\}
$$

be the "blow-up" of $G$ by $f$. If $\left\{\beta^{u}\right\}_{u \in G^{(0)}}$ is a full $f$-system, then we get a Haar system $\left\{\kappa^{z}\right\}_{z \in Z}$ on $G[Z]$ given by

$$
\kappa^{z}(f)=\int_{G} \int_{Z} f(z, g, w) d \beta^{s(g)}(w) d \lambda^{f(z)}(g) .
$$

The proof is relatively straightforward.

3.4. Imprimitivity groupoids. If $X$ is a free and proper right $G$-space, then assuming $G$ has open range and source maps, we can form the imprimitivity groupoid $G^{Z}$ as in [8, pp. 119ff.]. Specifically we let $G^{Z}$ be the quotient of $X *_{s} X=\{(x, y) \in$ $X \times X: s(x)=s(y)\}$ by the diagonal right $G$-action. Then $G^{Z}$ is a groupoid with respect to the operations $[x, y][y, z]=[x, z]$ and $[x, y]^{-1}=[y, z]$. If the moment map $r_{Z}: X \rightarrow G^{(0)}$ is open, then $X$ implements an equivalence between $G^{Z}$ and $G$. Again we can apply Theorem 2.1.

Proposition 3.7. Suppose that $G$ is a second countable, locally compact Hausdorff groupoid with a Haar system. Let $X$ be a free and proper right $G$-space with an open moment map. Then the imprimitivity groupoid $G^{Z}$ has a Haar system.

Remark 3.8. In [5, §§9-10], we associated a group $\operatorname{Ext}(G, \mathbf{T})$ to any second countable, locally compact Hausdorff groupoid G. In [5, Theorem 10.1] we showed that $\operatorname{Ext}(G, \mathbf{T})$ was naturally isomorphic to the Brauer group $\operatorname{Br}(G)$. The definition of $\operatorname{Ext}(G, \mathbf{T})$ required that we consider the space $\mathcal{P}(G)$ of all free and proper right $G$-spaces $X$ such that $G^{X}$ has a Haar system. In view of Proposition 3.7. $\mathcal{P}(G)$ becomes simply the collection of all free and proper right $G$-spaces. 


\section{ACKNOWLEDGEMENT}

The author would like to thank Marius Ionescu, Paul Muhly, Erik van Erp, Aidan Sims, and especially Jean Renault for helpful comments and discussions.

\section{REFERENCES}

[1] C. Anantharaman-Delaroche and J. Renault, Amenable groupoids, Monographies de L'Enseignement Mathématique [Monographs of L'Enseignement Mathématique], vol. 36, L'Enseignement Mathématique, Geneva, 2000. With a foreword by Georges Skandalis and Appendix B by E. Germain. MR:1799683 (2001m:22005)

[2] Étienne Blanchard, Déformations de $C^{*}$-algèbres de Hopf, Bull. Soc. Math. France 124 (1996), no. 1, 141-215. MR97f:46092

[3] Nicolas Bourbaki, Integration. II. Chapters 7-9, Elements of Mathematics (Berlin), SpringerVerlag, Berlin, 2004. Translated from the 1963 and 1969 French originals by Sterling K. Berberian. MR2098271 (2005f:28001)

[4] Rohit Dilip Holkar and Jean Renault, Hypergroupoids and $C$ *-algebras (English, with English and French summaries), C. R. Math. Acad. Sci. Paris 351 (2013), no. 23-24, 911-914, DOI 10.1016/j.crma.2013.11.003. MR3133603

[5] Alexander Kumjian, Paul S. Muhly, Jean N. Renault, and Dana P. Williams, The Brauer group of a locally compact groupoid, Amer. J. Math. 120 (1998), no. 5, 901-954. MR1646047 (2000b:46122)

[6] Ernest Michael, Continuous selections. I, Ann. of Math. (2) 63 (1956), 361-382. MR0077107 $(17,990 \mathrm{e})$

[7] Paul S. Muhly, Jean N. Renault, and Dana P. Williams, Equivalence and isomorphism for groupoid $C^{*}$-algebras, J. Operator Theory 17 (1987), no. 1, 3-22. MR873460 (88h:46123)

[8] Paul S. Muhly and Dana P. Williams, Groupoid cohomology and the Dixmier-Douady class, Proc. London Math. Soc. (3) 71 (1995), no. 1, 109-134, DOI 10.1112/plms/s3-71.1.109. MR 1327935 (97d:46082)

[9] Alan L. T. Paterson, Groupoids, inverse semigroups, and their operator algebras, Progress in Mathematics, vol. 170, Birkhäuser Boston, Inc., Boston, MA, 1999. MR 1724106 (2001a:22003)

[10] Arlan Ramsay, The Mackey-Glimm dichotomy for foliations and other Polish groupoids, J. Funct. Anal. 94 (1990), no. 2, 358-374. MR 1081649 (93a:46124)

[11] Jean Renault, A groupoid approach to $C^{*}$-algebras, Lecture Notes in Mathematics, vol. 793, Springer, Berlin, 1980. MR584266 (82h:46075)

[12] Jean Renault, Représentation des produits croisés d'algèbres de groupoïdes, J. Operator Theory 18 (1987), no. 1, 67-97. MR912813 (89g:46108)

[13] Jean Renault, Induced representations and hypergroupoids, SIGMA Symmetry Integrability Geom. Methods Appl. 10 (2014), Paper 057, 18, DOI 10.3842/SIGMA.2014.057. MR.3226993

[14] A. K. Seda, Haar measures for groupoids, Proc. Roy. Irish Acad. Sect. A 76 (1976), no. 5, 25-36. MR0427598(55 \#629)

[15] Anthony Karel Seda, On the continuity of Haar measure on topological groupoids, Proc. Amer. Math. Soc. 96 (1986), no. 1, 115-120. MR813822 (87m:46146)

[16] Jean-Louis Tu, Non-Hausdorff groupoids, proper actions and K-theory, Doc. Math. 9 (2004), 565-597 (electronic). MR2117427(2005h:22004)

[17] Jean-Louis Tu, Ping Xu, and Camille Laurent-Gengoux, Twisted K-theory of differentiable stacks (English, with English and French summaries), Ann. Sci. École Norm. Sup. (4) 37 (2004), no. 6, 841-910, DOI 10.1016/j.ansens.2004.10.002. MR2119241 (2005k:58037)

[18] Dana P. Williams, Crossed products of $C^{*}$-algebras, Mathematical Surveys and Monographs, vol. 134, American Mathematical Society, Providence, RI, 2007. MR2288954(2007m:46003)

Department of Mathematics, Dartmouth College, Hanover, New Hampshire 03755

E-mail address: dana.williams@dartmouth.edu 